\title{
Síndrome de Cushing exógeno por interacción medicamentosa de ritonavir y fluticasona inhalada. Reporte de tres casos pediátricos
}

\author{
Exogenous Cushing syndrome due to drug interaction of ritonavir and inhaled \\ fluticasone. Report of three pediatric cases
}

\author{
Dra. Lidia E. Torrado ${ }^{a}$ Dra. Analía Freire ${ }^{b}$ Dra. Mayra Martínez ${ }^{a}$ Dra. Ana Vieites ${ }^{b}$ Dr. Ignacio Bergadáb, \\ Dr. Eduardo L. López y y Dra. Aurelia A. Fallo
}

\begin{abstract}
RESUMEN
Elincremento dela expectativa de vida conel advenimiento dela terapia antirretroviral de alta eficacia plantea desafíos en cuanto a la toxicidad e interacciones medicamentosas. El síndrome de Cushing exógeno por interacción entre ritonavir y fluticasona inhalada en niños con diagnóstico de infección por virus de la inmunodeficiencia humana y patología pulmonar crónica es infrecuente. Hasta el momento, hay 20 casos reportados. Se describen 3 casos pediátricos con diagnóstico deinfección por virus de la inmunodeficiencia humana y patología pulmonar crónica que presentaron síndrome de Cushing exógeno con fluticasona inhalada en dosis habituales por la interacción medicamentosa entre esta y ritonavir. Los pacientes resolvieron el cuadro clínico luego de 2-4 meses de suspensión de la fluticasona y permanecieron asintomáticos en el seguimiento. Palabras clave: ritonavir, fluticasona, sindrome de Cushing, VIH.
\end{abstract}

\section{ABSTRACT}

The increase in life expectancy with the advent of highly effective antiretroviral therapy poses challenges in terms of toxicity and drug interactions. Exogenous Cushing syndrome by interaction between ritonavir and inhaled fluticasone in children diagnosed with human immunodeficiency virus infection and chronic pulmonary pathology is rare. So far, there are 20 cases reported. Three pediatric cases are reported, with a diagnosis of human immunodeficiency virus infection and chronic pulmonary pathology who presented exogenous Cushing syndrome with inhaled fluticasone at usual doses due to drug interaction between it and ritonavir. The patients resolved the clinical

a. Servicio de Infectología, Centro de Referencia de Sida Pediátrico, Hospital de Niños "Dr. Ricardo Gutiérrez", Buenos Aires, Argentina.

b. Centro de Investigaciones Endocrinológicas "Dr. César Bergadá" (CEDIE), Consejo Nacional de Investigaciones Científicas y Técnicas (CONICET), Fundación de Endocrinología Infantil (FEI), División de Endocrinología, Hospital de Niños "Dr. Ricardo Gutiérrez", Buenos Aires, Argentina.

Correspondencia:

Dra. Lidia E. Torrado: 1torrado@intramed.net

Financiamiento: Ninguno.

Conflicto de intereses: Ninguno que declarar.

Recibido: 17-2-2019

Aceptado: 5-11-2019 picture after 2-4 months of fluticasone suspension and remain asymptomatic in the follow-up.

Key words: ritonavir, fluticasone, Cushing syndrome, HIV.

http: / / dx.doi.org/10.5546/aap.2020.e278

Cómo citar: Torrado LE, Freire A, Martínez M, Vieites A, et al. Síndrome de Cushing exógeno por interacción medicamentosa de ritonavir y fluticasona inhalada. Reporte de tres casos pediátricos. Arch Argent Pediatr 2020;118(3):e278-e283.

\section{INTRODUCCIÓN}

En la última década, la ampliación global del tratamiento antirretroviral (TARV) de alta eficacia ha aumentado considerablemente la expectativa de vida de los pacientes infectados por el virus de la inmunodeficiencia humana (VIH) y ha disminuido las muertes relacionadas con el síndrome de inmunodeficiencia adquirida (sida). Pero el incremento en la expectativa de vida plantea desafíos con las comorbilidades, toxicidad e interacciones medicamentosas.

La enfermedad pulmonar crónica es prevalente en pacientes $\mathrm{VIH}+$, y los corticoides inhalados son de uso frecuente en ellos. El empleo concomitante de ritonavir y corticoides inhalados en dosis habituales puede dar lugar al síndrome de Cushing exógeno por bloquear la degradación del corticoide e incrementar sus concentraciones plasmáticas. ${ }^{1}$

Se describen 3 casos de síndrome de Cushing exógeno por interacción entre ritonavir y fluticasona inhalada en pacientes con diagnóstico de infección por VIH y patología pulmonar crónica.

\section{Descripción de casos}

Caso 1: Paciente masculino de 13 años con diagnóstico de infección por VIH de transmisión vertical. Recibía TARV con zidovudina, efavirenz, lopinavir/ritonavir con buena adherencia, evidenciada por carga viral $<200$ copias $/ \mathrm{ml}$ (<2,3 log.) y CD4+ del $27 \%$ (798 cél. $\left./ \mathrm{mm}^{3}\right)$. 
Por el diagnóstico de neumonía intersticial linfoide (NIL), inició fluticasona inhalada (500 $\mu \mathrm{g} /$ día). A los 60 días, presentó un incremento de peso de $6,7 \mathrm{~kg}$, facies redondeada, hipertricosis (Figura 1. A), giba dorsal leve, estrías horizontales rojo violáceo distribuidas en los miembros (Figura 1. B). Ante la sospecha de síndrome de Cushing exógeno, se solicitó cortisol sérico, que fue $<1 \mu \mathrm{g} / \mathrm{dl}$ (valor normal $-\mathrm{VN}-:>6 \mu \mathrm{g} / \mathrm{dl}$ ), y hormona adrenocorticotropa (adrenocorticotropic hormone; ACTH, por sus siglas en inglés) plasmática, que fue $<5 \mathrm{pg} / \mathrm{ml}$, lo que confirmó la supresión adrenal provocada por el corticoide exógeno. Se suspendió la fluticasona inhalada, y se dejaron pautas en caso de estrés grave para prevenir la insuficiencia adrenal. Luego de 30 días, se realizó cortisol sérico, que fue de 19,3 u/dl, y ACTH plasmática, de $26 \mathrm{pg} / \mathrm{ml}$. Estos niveles mostraron la recuperación de la respuesta hipofisaria y la normalización de la esteroidogénesis adrenal de cortisol. Cuatro meses después de la suspensión del corticoide inhalado, presentó la normalización del aspecto físico. Recibió como terapia alternativa montelukast (10 mg/día). En la actualidad, el paciente lleva 10 años de seguimiento, asintomático y fue transferido a un servicio de adultos.

Caso 2: Paciente masculino de 13 años de edad, con diagnóstico de infección por VIH de transmisión vertical. Recibía como TARV abacavir, didanosina y lopinavir / ritonavir, con buena adherencia evidenciada por carga viral < 34 copias / ml y CD4 del $25 \%$ (731 cél. / $\mathrm{mm}^{3}$ ). Presentaba el antecedente de tuberculosis miliar ya resuelta. Por la evolución a NIL, inició fluticasona (500 $\mu \mathrm{g} /$ día) + salmeterol inhalados. Luego de 1 año y 9 meses, presentó cambios físicos compatibles con síndrome de Cushing: cara de luna llena, acantosis nigricans en los pliegues e hipertricosis. Se realizó cortisol sérico matinal indetectable y cortisol libre urinario (CLU), que fue de $4 \mu \mathrm{g} / 24 \mathrm{~h}(\mathrm{VN}=20-90 \mu \mathrm{g} / 24 \mathrm{~h})$, lo que mostró una inadecuada producción integrada de cortisol y asociada a una supresión parcial de la respuesta hipofisaria $(A C T H: 11 \mathrm{pg} / \mathrm{ml})$. Se suspendió la fluticasona inhalada y, a los dos meses, se revirtió el aspecto físico cushingoide y presentó cortisol sérico de 19,5 $\mu \mathrm{g} / \mathrm{dl}$, CLU de $68 \mu \mathrm{g} / 24 \mathrm{~h}$ y $A C T H$ de $65 \mathrm{pg} / \mathrm{ml}$, los cuales confirmaron la normalización del eje adrenal. Actualmente, lleva 4 años de seguimiento, asintomático.

Caso 3: Paciente masculino de 7 años de edad, con diagnóstico de infección por VIH de transmisión vertical. Recibía TARV con zidovudina, lamivudina y lopinavir / ritonavir, con buena adherencia evaluada por carga viral $<34 \mathrm{c} / \mathrm{ml}(<1,53$ log. $)$ y CD4 del $31 \%$ $\left(1138\right.$ cél. $\left./ \mathrm{mm}^{3}\right)$. Por el diagnóstico de asma, inició el tratamiento con budesonida $(400 \mu \mathrm{g} /$ día) y salbutamol inhalados. Por el aumento del número de crisis, se rotó a fluticasona inhalada (500 $\mu \mathrm{g} /$ día). Luego de un mes del cambio, presentó aumento del apetito, incremento de $2 \mathrm{~kg}$ peso y fenotipo de síndrome de Cushing: facies redondeada y pletórica, aumento del perímetro abdominal e hipertricosis (Figura 2. A, B y C). Los niveles de cortisol sérico matinal $<1 \mu \mathrm{g} / \mathrm{dl}$ y $A C T H<5 \mathrm{pg} / \mathrm{ml}$ confirmaron la supresión del eje adrenal. Se suspendió la fluticasona y se

Figura 1. Cambios fenotípicos del síndrome de Cushing exógeno. Caso 1. A: hipertricosis en el dorso y el cuello. B: estrías rojas en ambos muslos

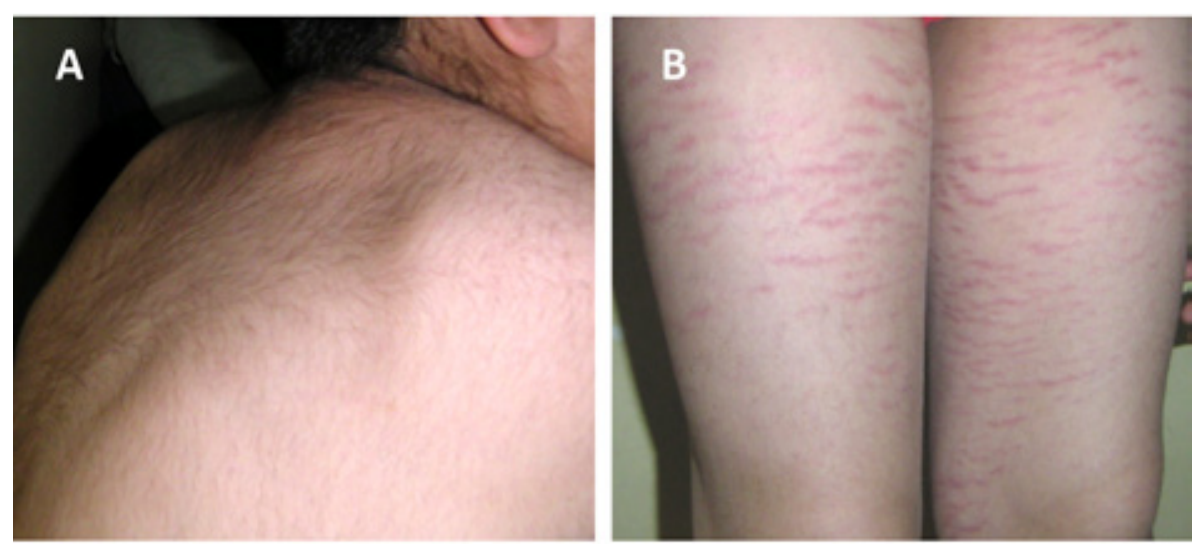


dieron las pautas de alarma ante la presencia de estrés grave o síntomas de insuficiencia adrenal. A los 45 días, presentó franca mejoría del aspecto físico y descenso de peso (Figura 2. D). Se realizó cortisol sérico, que fue de $27,9 \mu \mathrm{g} / \mathrm{dl}$, y ACTH, de $91 \mathrm{pg} / \mathrm{ml}$, lo que mostró indemnidad del eje adrenal. Continuó el tratamiento con montelukast (5 mg/día) con buena evolución clínica y de laboratorio. Actualmente, lleva 2 años de seguimiento asintomático.

\section{DISCUSIÓN}

En la infancia, generalmente, el síndrome de Cushing se debe a la administración exógena de glucocorticoides, los cuales son utilizados para trastornos inflamatorios, en altas dosis y por períodos prolongados. El tratamiento con fluticasona inhalada en dosis habituales no tiene un impacto negativo en el eje adrenal, ya que la biodisponibilidad sistémica es baja, menor del $1 \%$, y la degradación es rápida por medio del citocromo P450 (CYP450) hepático a través de la isoenzima 3A4. La familia del CYP450 humano comprende 57 isoenzimas, las cuales intervienen en el metabolismo normal de las hormonas esteroideas, pero también metabolizan alrededor de dos tercios de los medicamentos utilizados en humanos, principalmente, por 5 de esas isoenzimas (1A2, 2C9, 2C19, 2D6 y 3A4). ${ }^{2}$

Los pacientes tratados con inhibidores de

Figura 2. Caso 3. A: aspecto facial antes de iniciar el tratamiento con fluticasona. B y C: facies cushingoide y aspecto físico luego de un mes de interacción medicamentosa. C: Paciente luego de 45 días de suspensión de fluticasona, momento en el cual se constató la recuperación de la función adrenal

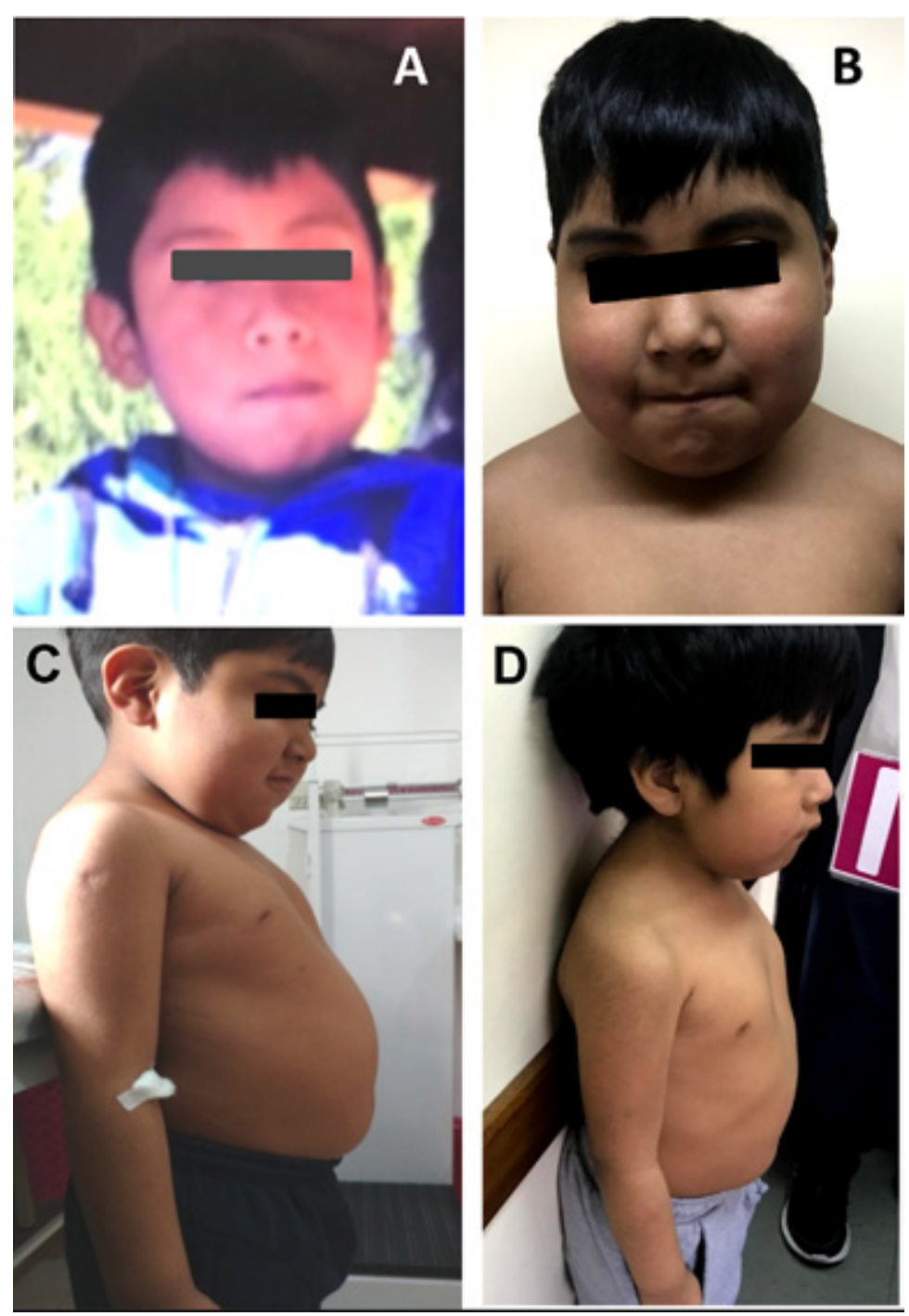


proteasa, en especial ritonavir, presentan una fuerte inhibición de este sistema enzimático, por lo cual las drogas que se metabolizan por esa vía se acumulan en forma exponencial. Dentro de los corticoides inhalados, la fluticasona es la de mayor vida media, mayor afinidad por el receptor, más lipofílica y de mayor volumen de distribución. Estas características farmacocinéticas hacen que, ante la inhibición de su degradación, se incremente su vida media y acumulación en los tejidos, por lo que es capaz de provocar síndrome de Cushing exógeno y supresión de la síntesis endógena adrenal de corticoides. ${ }^{3}$

En la Tabla 1, se presentan los 20 casos pediátricos de síndrome de Cushing exógeno secundario a la interacción de ritonavir con corticoides inhalados hallados en la literatura hasta diciembre de $2018^{4-13} \mathrm{y}$, al final, los tres pacientes aquí reportados para su comparación. La fluticasona fue el corticoide empleado en 20 de ellos, y la budesonida, en 3 casos. ${ }^{10}$ Esta última tiene menor potencia que la fluticasona, pero, por los casos revisados, la interacción medicamentosa parecería producirse en forma similar. ${ }^{10,14}$

Todos los pacientes presentaron el fenotipo de síndrome de Cushing con aumento progresivo de peso, facies de luna llena, aumento de la circunferencia abdominal, giba dorsal e hipertricosis. Todos recibían ritonavir en su esquema de base y un corticoide inhalado. La mediana de tiempo de coadministración, incluidos nuestros pacientes, hasta la detección del fenotipo Cushing fue 75 días (14-730).

En nuestros casos, como en la mayoría de los reportados, al momento del diagnóstico, se confirmó la inhibición del eje mediante los niveles disminuidos o indetectables de $A C T H$ y de cortisol sérico. El corticoide exógeno acumulado por la inhibición de su metabolismo/ degradación inhibe el eje a nivel hipotalámico e hipofisario por retroalimentación negativa. Esto puede dar lugar a cuadros de insuficiencia adrenal, como se halló en algunos casos de la literatura. ${ }^{5,7}$ Ninguno de nuestros pacientes presentó un cuadro de insuficiencia adrenal, pero, en todos ellos, se dieron pautas de alarma frente a una urgencia con indicaciones precisas de administración parenteral de corticoides. A su vez, debe considerarse también que la excesiva y prolongada exposición a corticoides puede producir retraso de crecimiento, pérdida del contenido mineral óseo y alteraciones en el sistema inmune.

Nuestros 3 casos fueron varones, aunque, en la bibliografía, se reporta un predominio de mujeres $(2: 1)$. La edad de estos pacientes fue de 7 y 13 años (2 casos). En la literatura, la edad osciló entre 1 y 16 años. Nuestros pacientes lograron la resolución clínica entre 1,5 y 4 meses luego de haberse suspendido el corticoide al igual que la mayoría de los reportados previamente (excepto 3 casos a los 6 meses). No se observó relación o diferencias de importancia entre las dosis de ritonavir o fluticasona entre los casos de la literatura y los aquí reportados. No se reportó ningún fallecimiento asociado a dicha interacción farmacológica.

En los pacientes en TARV con ritonavir, solo o como booster de otro inhibidor de proteasa, que presenten patología pulmonar crónica, se podrían usar antagonistas de los receptores de los leucotrienos (montelukast) o agonistas $\beta_{2} \cdot{ }^{10}$ En el caso de requerir corticoides inhalados, el recomendado sería la beclometasona, dado su escaso metabolismo a través del sistema enzimático CYP. ${ }^{15}$ Otros fármacos de uso habitual utilizan los mismos mecanismos de degradación, entre ellos: rifampicina, ergotamina, tramadol, midazolam, ciclosporina, posaconazol e itraconazol. Es importante conocer el riesgo de estas interacciones medicamentosas, que podrían traer consecuencias serias e incluso poner en riesgo la vida, para evitarlas e interpretar adecuadamente el cuadro clínico.

\section{REFERENCIAS}

1. Barragan P, PodzamczerD. Lopinavir/ ritonavir: a protease inhibitor for HIV-1 treatment. Expert Opin Pharmacother. 2008; 9(13):2363-75.

2. Gong Y, Haque S, Chowdhury P, Cory TJ, et al. Pharmacokinetics and pharmacodynamics of cytochrome P450 inhibitors for HIV treatment. Expert Opin Drug Metab Toxicol. 2019; 15(5):417-27.

3. SaberiP,Phengrasamy T,NguyenDP.Inhaled corticosteroid use in HIV-positive individuals taking protease inhibitors: a review of pharmacokinetics, case reports and clinical management. HIV Med. 2013; 14(9):519-29.

4. Johnson SR, Marion AA, Vrchoticky T, Emmanuel PJ, et al. Cushing syndrome with secondary adrenal insufficiency from concomitant therapy with ritonavir and fluticasone. J Pediatr. 2006; 148(3):386-8.

5. Arrington-Sanders R, Hutton N, Siberry GK. Ritonavirfluticasone interaction causing Cushing syndrome in HIVinfected children and adolescents. Pediatr Infect Dis J. 2006; 25(11):1044-8.

6. St Germain RM, Yigit S, Wells L, Girotto J, et al. Cushing syndrome and severe adrenal suppression caused by fluticasone and protease inhibitor combination in an HIV-infected adolescent. AIDS Patient Care STDS. 2007; 21(6):373-7.

7. Pessanha TM, Campos JM, Barros AC, Pone MV, et al. Iatrogenic Cushing's syndrome in an adolescent with AIDSs on ritonavir and inhaled fluticasone. Case report and literature review. AIDS. 2007;21(4):529-32. 
e282 / Arch Argent Pediatr 2020;118(3):e278-e283 / Presentación de casos clínicos

TABLA 1. Casos pediátricos del presente estudio y reportados en la literatura (2006-2018) de síndrome de Cushing por interacción ritonavir-fluticasona/budesonida inhalada o intranasal

\begin{tabular}{|c|c|c|c|c|c|c|c|c|c|}
\hline $\begin{array}{l}\text { Caso n. }{ }^{\circ} \\
\text { (ref.) }\end{array}$ & $\begin{array}{l}\text { Sexo; } \\
\text { edad (años) }\end{array}$ & TARV & $\begin{array}{c}\text { Dosis de } \\
\text { ritonavir /día } \\
\text { (mg) }\end{array}$ & $\begin{array}{c}\text { Dosis } \\
\text { diaria de } \\
\text { corticoide } \\
\text { IH/IN }(\mu \mathrm{g})\end{array}$ & $\begin{array}{l}\text { Duración de } \\
\text { la terapia } \\
\text { combinada } \\
\text { (días) }\end{array}$ & $\begin{array}{l}\text { Recuento CD4 } \\
\left(\mathrm{c} / \mathrm{mm}^{3}\right) / \mathrm{CV} \\
(\text { cop. } / \mathrm{ml} \\
\text { o log.) }\end{array}$ & $\begin{array}{c}\text { Niveles de } \\
\text { cortisol/ACTH } \\
\text { plasmáticos }\end{array}$ & $\begin{array}{cc}\text { Estrategia } & \\
\text { terapéutica } & \mathbf{r} \\
& \\
& \text { sn } \\
& \end{array}$ & $\begin{array}{c}\text { Tiempo de } \\
\text { resolución del } \\
\text { sme. de Cushing } \\
\text { (meses) }\end{array}$ \\
\hline$\overline{1^{(4)}}$ & $\mathrm{F} ; 12$ & $\begin{array}{l}\text { 3TC, AZT } \\
\text { LPN / RTV }\end{array}$ & 134 & $\begin{array}{c}\text { Fluticasona } \\
1000 \mathrm{IH}\end{array}$ & 60 & SD & Indetectables & $\begin{array}{c}\text { Rotación de } \\
\text { LPN / RTV } \\
\text { a EFV } \\
\end{array}$ & 6 \\
\hline $2^{(4)}$ & $\mathrm{F} ; 15$ & $\begin{array}{l}\text { LPN / RTV } \\
\text { 3TC, ABC }\end{array}$ & 200 & $\begin{array}{c}\text { Fluticasona } \\
1000 \mathrm{IH}\end{array}$ & 90 & SD & Indetectables & $\begin{array}{l}\text { Suspensión } \\
\text { fluticasona }\end{array}$ & 2,5 \\
\hline $3^{(5)}$ & $\mathrm{F} ; 11,4$ & $\begin{array}{l}\text { LPN / RTV } \\
\text { D4T, 3TC }\end{array}$ & 133 & $\begin{array}{l}\text { Fluticasona } \\
\text { 220IH }\end{array}$ & SD & $1161 /<50$ & Indetectables & $\begin{array}{l}\text { Suspensión } \\
\text { fluticasona } \\
\text { Rotación a EFV, } \\
\text { ddI, FTC } \\
\end{array}$ & 3 \\
\hline$\overline{4^{(5)}}$ & M; 9,5 & $\begin{array}{l}\mathrm{LPN} / \mathrm{RTV}, \\
\text { 3TC, TDF }\end{array}$ & 133 & $\begin{array}{c}\text { Fluticasona } \\
220 \mathrm{IH}\end{array}$ & SD & $461 / 1670$ & Indetectables & $\begin{array}{l}\text { Suspensión } \\
\text { fluticasona }\end{array}$ & 3 \\
\hline$\overline{5^{(5)}}$ & $\mathrm{F} ; 1,8$ & $\begin{array}{l}\text { LPN / RTV } \\
3 \mathrm{TC}, \mathrm{d} 4 \mathrm{~T}\end{array}$ & 60 & $\begin{array}{c}\text { Fluticasona } \\
220 \mathrm{IH}\end{array}$ & 180 & $1712 /<50$ & Indetectables & $\begin{array}{l}\text { Suspensión } \\
\text { fluticasona }\end{array}$ & 4 \\
\hline $6^{(5)}$ & $\mathrm{F} ; 16,8$ & $\begin{array}{c}\mathrm{LPN} / \mathrm{RTV}, \\
\mathrm{ABV}, \mathrm{AZT} \\
\text { 3TC }\end{array}$ & 200 & $\begin{array}{l}\text { Fluticasona } \\
250 \mathrm{IH}\end{array}$ & 194 & SD & SD & $\begin{array}{l}\text { Suspensión } \\
\text { fluticasona }\end{array}$ & 3 \\
\hline$\overline{7^{(6)}}$ & $\mathrm{F} ; 14$ & $\begin{array}{l}\text { ddI, TDF } \\
\text { RTV, ATV }\end{array}$ & 100 & $\begin{array}{l}\text { Fluticasona } \\
500 \mathrm{IH}\end{array}$ & 14 & $\begin{array}{c}664 / \log . \\
<2,6\end{array}$ & Indetectables & $\begin{array}{c}\text { Suspensión } \\
\text { fluticasona } \\
\text { Rotar a albuterol + } \\
\text { inicio ARL } \\
\text { Suspende } \\
\text { RTV y ATV } \\
\end{array}$ & + \\
\hline$\overline{8^{(7)}}$ & $\mathrm{F} ; 16$ & $\begin{array}{l}\text { 3TC, d4T } \\
\text { RTV } \\
\end{array}$ & SD & $\begin{array}{c}\text { Fluticasona } \\
500 \mathrm{IH}\end{array}$ & 90 & SD & Indetectables & $\begin{array}{c}\text { Rotación } \\
\text { de RTV a EFV }\end{array}$ & 2 \\
\hline$\overline{9^{(8)}}$ & M; 9 & $\begin{array}{c}\text { ddI, 3TC } \\
\text { LPN/RTV }\end{array}$ & 100 & $\begin{array}{c}\text { Fluticasona } \\
440 \mathrm{IH} \\
\end{array}$ & 60 & $-/ 75$ & Indetectables & $\begin{array}{c}\text { Descenso } \\
\text { fluticasona }\end{array}$ & 1,5 \\
\hline $10^{(9)}$ & $\mathrm{F} ; 9$ & $\begin{array}{c}\text { LPN/RTV } \\
\text { ddI, 3TC }\end{array}$ & SD & $\begin{array}{c}\text { Fluticasona } \\
250 \mathrm{IH}\end{array}$ & SD & SD & SD & SD & SD \\
\hline$\overline{11^{(9)}}$ & $\mathrm{F} ; 10$ & $\begin{array}{c}\mathrm{LPN} / \mathrm{RTV} \\
\mathrm{ABC}\end{array}$ & SD & $\begin{array}{c}\text { Fluticasona } \\
1000 \mathrm{IH} \\
\end{array}$ & SD & SD & SD & SD & SD \\
\hline $12^{(9)}$ & M; 16 & $\begin{array}{c}\text { FosAPV, RTV } \\
\text { AZT, TDF }\end{array}$ & SD & $\begin{array}{c}\text { Fluticasona } \\
500 \mathrm{IH} \\
\end{array}$ & SD & SD & SD & SD & SD \\
\hline$\overline{13^{(10)}}$ & $\mathrm{F} ; 4$ & RTV & $300 / \mathrm{m}^{2}$ & $\begin{array}{l}\text { Budesonida } \\
1200 \mathrm{IH} / \mathrm{IN}\end{array}$ & 90 & $-/<50$ & SD & $\begin{array}{c}\text { Rotación } \\
\text { de RTV a EFV } \\
\text { Suspensión } \\
\text { budesonida }\end{array}$ & 1 \\
\hline $14^{(10)}$ & $\mathrm{F} ; 4$ & LPN/RTV & $235 / \mathrm{m}^{2}$ & $\begin{array}{l}\text { Budesonida } \\
200 \mathrm{IH}\end{array}$ & 730 & $-/<50$ & SD & $\begin{array}{c}\text { Rotación de } \\
\text { LPN/RTV a EFV }\end{array}$ & 2 \\
\hline $15^{(10)}$ & $\mathrm{M} ; 7$ & SD & SD & $\begin{array}{c}\text { Budesonida } \\
200 \mathrm{IH} \\
\end{array}$ & 60 & $-/<50$ & SD & $\begin{array}{l}\text { Suspensión } \\
\text { Budesonida }\end{array}$ & 1,5 \\
\hline $16^{(11)}$ & $\mathrm{F} ; 12$ & $\begin{array}{l}\text { 3TC, AZT } \\
\text { LPV / RTV }\end{array}$ & 200 & $\begin{array}{l}\text { Fluticasona } \\
200 \mathrm{IH}\end{array}$ & 730 & $-/<40$ & Indetectables & $\begin{array}{c}\text { Descenso } \\
\text { fluticasona } \\
\text { Inicio ARL } \\
\end{array}$ & 3 \\
\hline $17^{(12)}$ & $\mathrm{M} ; 7$ & $\begin{array}{l}\text { 3TC, AZT } \\
\text { LPN/RTV }\end{array}$ & SD & $\begin{array}{c}\text { Fluticasona } \\
500 \mathrm{IH}\end{array}$ & 180 & SD & SD & $\begin{array}{l}\text { Suspensión } \\
\text { fluticasona }\end{array}$ & 6 \\
\hline $18^{(12)}$ & M; 7 & $\begin{array}{l}\text { AZT, 3TC } \\
\text { LPN/RTV }\end{array}$ & SD & $\begin{array}{l}\text { Fluticasona } \\
200 \text { IN }\end{array}$ & 60 & SD & Indetectables & $\begin{array}{c}\text { Suspensión } \\
\text { fluticasona } \\
\text { Rotación a } \\
\text { budesonida }\end{array}$ & SD \\
\hline $19^{(12)}$ & $\mathrm{F} ; 14$ & $\begin{array}{l}\text { AZT, 3TC } \\
\text { LPN/RTV }\end{array}$ & SD & $\begin{array}{l}\text { Fluticasona } \\
500 \mathrm{IH}\end{array}$ & 30 & SD & Indetectables & $\begin{array}{c}\text { Descenso } \\
\text { fluticasona } \\
\text { Rotación de } \\
\text { LPN/RTV a EFV }\end{array}$ & 6 \\
\hline $20^{(13)}$ & $\mathrm{F} ; 6$ & $\begin{array}{l}\text { LPN / RTV } \\
3 T C / A B C\end{array}$ & 80 & $\begin{array}{l}\text { Fluticasona } \\
200 \text { IN }\end{array}$ & 60 & SD & Indetectables & $\begin{array}{l}\text { Descenso } \\
\text { fluticasona }\end{array}$ & 3 \\
\hline $21(*)$ & M; 13 & $\begin{array}{l}\text { AZT, 3TC, } \\
\text { LPN/RTV }\end{array}$ & 200 & $\begin{array}{l}\text { Fluticasona } \\
500 \mathrm{IH}\end{array}$ & 60 & $798 /<200$ & Indetectables & $\begin{array}{l}\text { Suspensión } \\
\text { fluticasona } \\
\text { Inicio ARL } \\
\end{array}$ & 4 \\
\hline $22(*)$ & M; 13 & $\begin{array}{l}\text { ABC, 3TC, } \\
\text { LPN/RTV }\end{array}$ & 200 & $\begin{array}{l}\text { Fluticasona } \\
500 \mathrm{IH}\end{array}$ & 700 & $731 /<34$ & $\begin{array}{l}\text { Indetectable/ } \\
11 \mathrm{pg} / \mathrm{ml}\end{array}$ & $\begin{array}{l}\text { Suspensión } \\
\text { fluticasona } \\
\text { Inicio ARL } \\
\end{array}$ & 2 \\
\hline $23(*)$ & M; 7 & $\begin{array}{l}\text { AZT, 3TC, } \\
\text { LPN/RTV }\end{array}$ & 100 & $\begin{array}{l}\text { Fluticasona } \\
500 \mathrm{IH}\end{array}$ & 36 & $1138 /<34$ & Indetectables & $\begin{array}{l}\text { Suspensión } \\
\text { fluticasona } \\
\text { Inicio ARL }\end{array}$ & 1,5 \\
\hline
\end{tabular}

TARV: terapia antirretroviral; LPN: lopinavir; RTV: ritonavir; 3TC: lamivudina; ABC: abacavir; ATV: atazanavir;

AZT: zidovudina; d4T: estavudina; ddI: didanosina; EFV: efavirenz; FPV: fosamprenavir; TDF: tenofovir; CV: carga viral;

ARL: antagonistas de los receptores de leucotrienos; ACTH: hormona estimulante del corticotropo; SD: sin datos; IH: inhalado;

IN: intranasal.

$\left.{ }^{*}\right)$ Pacientes de este reporte de casos. 
8. Bhumbra NA, Sahloff EG, Oehrtman SJ, Horner JM. Exogenous Cushing syndrome with inhaled fluticasone in a child receiving lopinavir/ ritonavir. Ann Pharmacother. 2007; 41(7):1306-9.

9. Daveluy A, Raignoux C, Miremont-Salame G, Girodet PO, et al. Drug interactions between inhaled corticosteroids and enzymatic inhibitors. Eur J Clin Pharmacol. 2009; 65(7):7435.

10. Gray D, Roux P, Carrihill M, Klein M. Adrenal suppression and Cushing's syndrome secondary to ritonavir and budesonide. S Afr Med J. 2010; 100(5):296-7.

11. Mahlab-Guri K, Asher I, Gradstein S, Zung A, et al. Inhaled fluticasone causes iatrogenic Cushing's syndrome in patients treated with Ritonavir. J Asthma. 2011;48(8):860-3.

12. Aguirre M, GutiérrezS, QuianJ, Ramírez MJ, etal.Síndrome de Cushing secundario a interacción medicamentosa ritonavir/fluticasona. Arch Pediatr Urug. 2012; 83(1):21-5.

13. Van den Berg S, Van $\square$ t Veer NE, Emmen JMA, Van Beek RHT. Fluticasone furoate induced iatrogenic Cushing syndrome in a pediatric patient receiving anti-retroviral therapy. Endocrinol Diabetes Metab Case Rep. 2017; 2017:160158.

14. Kedem E, Shahar E, Hassoun G, Pollack S. Iatrogenic Cushing's syndrome due to coadministration of ritonavir and inhaled budesonide in an asthmatic human immunodeficiency virus infected patient. J Asthma. 2010; 47(7):830-1.

15. Foisy MM, Yakiwchuk EMK, Chiu I, Singh AE. Adrenal suppression and Cushing's syndrome secondary to an interaction between ritonavir and fluticasone: a review of the literature. HIV Med. 2008; 9(6):389-96. 